\title{
Éditorial
}

\section{Innover autour de la fonction d'enseignant-ressource : entre recherche, théorie et pratique}

Auteurs

Nancy Granger, Université de Sherbrooke, Canada, nancy.granger@usherbrooke.ca

Philippe Tremblay, Université Laval, Canada, philippe.tremblay@fse.ulaval.ca

Philippe Maloney, Centre éducatif l'Abri, Canada, philippe.maloney@csdufer.qc.ca 


\section{$\&$}

\section{REVUE HYBRIDE DE L'ÉDUCATION}

Depuis plusieurs années, le coenseignement entre un enseignant titulaire de classe et un enseignant-ressource ${ }^{1}$ est devenu une réalité de plus en plus partagée dans les écoles secondaires du Québec (Tremblay et Granger, 2020). Comme il s'agit d'une fonction relativement nouvelle, peu de recherches viennent éclairer l'organisation de cette ressource ainsi que les conditions de sa mise en œuvre effective dans les différents milieux. Des études récentes qui découlent d'un questionnaire québécois (Granger et Tremblay, 2019 ; Tremblay et Granger, 2020) qui avait pour but de dresser un état des lieux de la fonction d'enseignant-ressource montrent que ce sont $45 \%$ des enseignants-ressources qui disent avoir vécu une situation de coenseignement en classe où leur rôle consistait à assister l'enseignant dans la classe. Ils rapportent alors avoir apprécié le partage et la collaboration avec une collègue et témoignent s'être sentis utiles autant pour les élèves que pour l'enseignant. Ce dispositif demande toutefois d'appréhender autrement l'organisation des services à l'élève, les rôles des divers acteurs scolaires, mais aussi de réfléchir aux valeurs qui soustendent ce service. Selon, les observations réalisées dans des contextes scientifique ou pratique, il convient de souligner l'intérêt de plusieurs équipes-écoles au fil des années à optimiser le rôle de l'enseignantressource (Granger 2012 ; Dubé, Granger et Dufour, 2015; Granger et Dubé, 2015 ; Granger et Tremblay, 2019 ; Tremblay et Granger, 2020).

Parmi les dispositifs pertinents, l'utilisation du coenseignement et de pratiques innovantes ou probantes destinées aux élèves les plus en difficulté constituent une alternative à la co-intervention externe davantage associée à un modèle ségrégatif. Tremblay (2012, p.71) définit le coenseignement "comme un travail pédagogique en commun, dans un même groupe et dans un même temps, de deux ou de plusieurs enseignants se partageant les responsabilités éducatives pour atteindre les objectifs spécifiques. Cette collaboration peut fonctionner à temps partiel ou à temps complet ». Le coenseignement offre l'avantage de réduire le ratio enseignant/élèves pour permettre aux premiers d'interagir plus souvent avec les élèves en difficulté et leur fournir un enseignement plus individualisé et intensif (Friend et Cook, 2007), tout en étant moins stigmatisant (Murawski et Hughes, 2009). Le coenseignement est ainsi

\footnotetext{
${ }^{1}$ Depuis 2007, au Québec, en enseignement secondaire, existe la fonction d'enseignantsressource (ER) pour soutenir les élèves en difficulté intégrés en classe ordinaire. L'ER est libéré pour un maximum de $50 \%$ de sa tâche éducative pour exercer ses fonctions. Cinq missions lui sont assignés. Auprès des élèves en difficulté, l'ER offre un accompagnement personnalisé et assume un rôle de suivi scolaire et d'aide et des tâches d'encadrement auprès de ces élèves. L'ER travaille en concertation avec les enseignantes et enseignants responsables des élèves en difficulté qui lui sont adressés en portant une attention particulière aux enseignantes et enseignants en début de carrière. II travaille également en concertation avec les autres intervenantes et intervenants qui œuvrent auprès des élèves: psychoéducateurs, psychologues, travailleurs sociaux, techniciennes en éducation spécialisée, etc.
} 


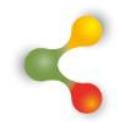

\section{REVUE HYBRIDE DE L'ÉDUCATION}

étroitement associé à une conception non pas corrective, mais qualitative de l'intervention auprès des élèves en difficulté.

Concrètement, dans les écoles secondaires, ce coenseignement peut s'organiser de manière intensive (p.ex. en présence à $50 \%$ ou plus dans un groupe ciblé), de manière stratégique (p.ex. dédié au rehaussement des compétences en lecture-écriture, à l'utilisation des outils technologiques, au suivi d'élèves ciblés) ou de manière dispersée (selon les demandes des enseignants, lors de projets particuliers). On observe de plus que les enseignants-ressources qui coenseignent peuvent collaborer avec plusieurs enseignants de leur école. Le temps nécessaire à la coplanification ou la concertation constitue d'ailleurs un enjeu à considérer dans la tâche des coenseignants.

Ce numéro spécial de la Revue hybride de l'éducation (RHÉ) fait suite au symposium de l'ACFAS 2018 portant sur les "Pratiques innovantes autour de la fonction d'enseignant-ressource». À cette occasion, chercheurs, enseignants, directions d'établissement, conseillers pédagogiques s'étaient réunis pour présenter des pratiques novatrices, des projets, des modes d'organisation singuliers autour de la fonction d'enseignant-ressource. Cet ouvrage collectif se veut un lieu de rencontre entre la théorie et la pratique afin de promouvoir les pratiques inclusives et novatrices qui ont démontré leur pertinence au sein de la fonction d'enseignant-ressource à l'ordre secondaire. II vise la mise en commun d'expertises complémentaires pour aborder ce sujet encore peu exploré et a pour but de mettre en lumière le rôle de chacun des acteurs et les défis à relever dans un souci d'arrimage des services à l'élève.

Les articles de ce numéro s'inscrivent autour de trois axes, soit : 1) Les aspects théoriques de la recherche ; 2 ) Les pratiques de gestion autour de l'organisation des services, et 3) Les pratiques de coenseignement et pratiques innovantes au sein de la classe.

Dans le premier axe, trois articles présentent des résultats de recherche. Le premier texte de Nancy Granger (Université de Sherbrooke) et Philippe Tremblay (Université Laval) aborde les effets du dialogue cogénératif en contexte d'accompagnement au coenseignement en enseignement secondaire au Québec. Des verbatims et des notes d'observations en contexte de coenseignement ont été analysés. L'objectif était de savoir comment les types de dialogue cogénératif s'opérationnalisent chez les coenseignants et comment il peut servir à l'accompagnateur pour analyser les contenus des concertations pour la recherche et pour ensuite guider les coenseignants dans l'action. Le deuxième article de Patrice Bourdon, maitre de conférences à l'Université de Nantes, porte sur les ressources et collaborations pour faciliter la scolarisation inclusive dans un établissement scolaire du $2^{\mathrm{e}}$ degré à Nantes en France. L'auteur analyse les différentes fonctions qui font « ressources » et les collaborations conséquentes mises en œuvre en appui de la 


\section{$\&$}

\section{REVUE HYBRIDE DE L'ÉDUCATION}

scolarisation pour un public à besoins éducatifs particuliers. Dans son texte, Patrice Bourdon met en lumière à quel point l'organisation scolaire est intimement liée la fonction de ressource. Qu'elles soient formelles ou informelles, les interventions sont tributaires de la prise en compte des besoins de chacun au sein du système ou de l'institution. Le troisième et dernier texte de cet axe est celui de Gilles Moreau et Marie Toullec, également de l'université de Nantes. Ils décrivent les mécanismes didactiques en jeu dans une situation de coenseignement en milieu inclusif, où un enseignant de mathématiques et une enseignante spécialisée se partagent, au sein de la même classe, la responsabilité des apprentissages. Les auteurs tentent de comprendre si ce coenseignement favorise et améliore les apprentissages, et de quelle manière.

Dans le deuxième axe portant sur les pratiques de gestion autour de l'organisation des services à l'élève. Deux articles éclairent les modalités de mise en œuvre du coenseignement et renseignent sur le leadership à exercer dans le pilotage de ce dispositif. L'article de Philippe Maloney, directeur du Centre éducatif l'Abri de Port-Cartier à la Commission scolaire du Fer, porte sur le coenseignement comme dispositif pour soutenir l'inclusion scolaire. II dresse un bilan de l'accompagnement reçu et de la démarche collaborative amorcée pour optimiser l'organisation des services dans cette école. L'article de Nathalie Deslauriers et Maxime CoulombeGodbout, directeurs de l'école de l'Odyssée à la Commission scolaire des Affluents, relate de quelle façon ils ont dû composer avec les multiples facettes de la fonction d'enseignant-ressource en vue de choisir un dispositif qui convienne à la fois aux besoins des élèves, des enseignants et des balises imposées par la grille horaire. Ces deux équipes de directions d'établissement mettent en lumière le rôle important de facilitateur qu'ils ont joué.

Enfin, dans le dernier axe, il est question des pratiques de coenseignement et pratiques innovantes au sein de la classe, Stéphane Maltais et Yves Daigneault, enseignants de $3^{\mathrm{e}}$ secondaire, présentent le groupe "Stratégies Plus» créé à l'école secondaire de l'Odyssée à la Commission scolaire des Affluents. Ce groupe réunit divers intervenants du milieu scolaire afin de soutenir les élèves qui ont des difficultés en mathématiques et en français. Animé par la volonté de "faire autrement », il privilégie le coenseignement et la concertation entre les participants. Sa mise en place a entrainé de nombreux changements dans l'organisation et les pratiques scolaires. De leur côté, Nadia Gray et Julie Jeannotte, font état de leur expérience de coenseignement en classe de français à des groupes d'élèves à risque à l'école Louis-Philippe-Paré de la Commission scolaire des Grandes Seigneuries. Elles décrivent les outils, les mesures et les méthodes de travail qu'elles ont expérimentées et jugent efficaces pour soutenir les élèves. Les conditions à réunir pour une saine collaboration entre coenseignants sont aussi abordées. Enfin, Éric Chatigny enseignant à l'école secondaire Curé-Mercure de la Commission scolaire des Laurentides s'intéresse au processus de nomination de 


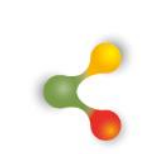

\section{REVUE HYBRIDE DE L'ÉDUCATION}

l'enseignant-ressource. II est d'abord question d'une démarche d'analyse des besoins des élèves, puis d'un processus de sélection invitant les enseignants à faire valoir leurs compétences professionnelles au regard de la fonction à occuper et des besoins à combler. Cette façon de procéder met en lumière la nécessité de démontrer un niveau de compétence élevé quant au soutien à apporter aux élèves, mais aussi aux collègues enseignants.

Bonne lecture! 


\section{REVUE HYBRIDE DE L'ÉDUCATION}

\section{Références}

Dubé, F. Granger, N. et Dufour, F. (2015). Continuing Education for High School Resource Teachers and Their Sense of Self-efficacy. American Journal of Educational Research. 3(6), 707-712.

Friend, M. P. et Cook, L. (2007). Interactions : Collaboration skills for school professionals. Longman.

Granger, N. (2012). La transformation des pratiques professionnelles chez les enseignants du secondaire : analyse des effets de la participation aux cercles d'apprentissage et d'inclusion (Thèse de doctorat inédite). Sherbrooke, QC : Université de Sherbrooke.

Granger, N. et Dubé, F. (2015). Définir la fonction d'enseignant-ressource dans le secondaire: une recherche-action formation. La nouvelle revue de l'adaptation et de la scolarisation, 2(70-71), 121-136.

Granger, N. et Tremblay, P. (2019). Satisfaction des enseignantsressources à l'égard des rôles et des fonctions pour soutenir la réussite des élèves à besoins spécifiques. McGill Journal of Education, 54, 132-150.

Murawski, W. W. et Hughes, C. E. (2009). Response to intervention, collaboration and co-teaching: a logical combination for successful systemic change. Preventing School Failure: Alternative Education for Children and Youth, 53(4), 267-277.

Tremblay, P. (2012). Inclusion scolaire ( $2^{\mathrm{e}}$ édition). Dispositifs et pratiques pédagogiques. De Boeck.

Tremblay, P. et Granger, N. (accepté). Portrait de la fonction d'enseignantressource au Québec: quels services pour quels élèves. Formation et profession. 